\title{
A Quiet Standing Index for Testing the Postural Sway of Healthy and Diabetic Adults Across a Range of Ages
}

\author{
Robert J. Schilling*, Senior Member, IEEE, Erik M. Bollt, George D. Fulk, Joseph D. Skufca, \\ Ahmad F. Al-Ajlouni, Member, IEEE, and Charles J. Robinson, Fellow, IEEE
}

\begin{abstract}
A quietstanding index is developed for tracking the postural sway of healthy and diabetic adults over a range of ages. Several postural sway features are combined into a single composite feature $C$ that increases with age $a$. Sway features are ranked based on the $r^{2}$-values of their linear regression models, and the composite feature is a weighted sum of selected sway features with optimal weighting coefficients determined using principal component analysis. A performance index based on both reliability and sensitivity is used to determine the optimal number of features. The features used to form $C$ include power and distance metrics. The quiet standing index is a scalar that compares the composite feature $C$ to a linear regression model $f(a)$ using $C^{\prime}(a)=C / f(a)$. For a motionless subject, $C^{\prime}=0$, and when the composite feature exactly matches the healthy control $(\mathrm{HC})$ model, $C^{\prime}=1$. Values of $C^{\prime} \gg 1$ represent excessive postural sway and may indicate impaired postural control. Diabetic neurologically intact subjects, nondiabetic peripheral neuropathy subjects $(\mathrm{PN})$, and diabetic PN subjects (DPN) were evaluated. The quiet standing indexes of the PN and DPN groups showed statistically significant increases over the HC group. Changes in the quiet standing index over time may be useful in identifying people with impaired balance who may be at an increased risk of falling.
\end{abstract}

Index Terms-Diabetes, peripheral neuropathy ( $\mathrm{PN})$, postural sway metrics, quiet standing index.

\section{INTRODUCTION}

$\mathbf{T}$ HE CONTROL of balance is a key aspect of mobility over the human lifespan from young children learning to stand

Manuscript received November 11, 2007; revised March 15, 2008. First published August 12, 2008; current version published March 25, 2009. This work was supported in part by a Veterans Administration (VA) Rehabilitation Research and Development (R\&D) under Grant E2143PC and a VA Senior Rehabilitation Research Career Scientist Award to Columbia Journalism Review (CJR), in part by the National Institutes of Health (NIH) under Grant R01AG026553, and in part by a Coulter Foundation endowment to Clarkson University. Asterisk indicates corresponding author.

*R. J. Schilling is with the Department of Electrical and Computer Engineering, Clarkson University, Potsdam, NY 13699 USA (e-mail: schillin@clarkson.edu).

E. M. Bollt and J. D. Skufca are with the Department of Mathematics and Computer Science, Clarkson University, Potsdam, NY 13699 USA (e-mail: bolltem@clarkson.edu; jskufca@clarkson.edu).

G. D. Fulk is with the Department of Physical Therapy, Clarkson University, Potsdam, NY 13699 USA.

A. F. Al-Ajlouni is with Hijjawi Faculty of Engineering Technology, Department of Communication Engineering, Yarmouk University, Irbid 71100-71115, Jordan.

C. J. Robinson is with the Syracuse Veterans Administration (VA) Medical Center, Syracuse, NY 13210 USA, and also with the Center for Rehabilitation Engineering, Science and Technology (CREST), Clarkson University, Potsdam, NY 13699 USA.

Color versions of one or more of the figures in this paper are available online at http://ieeexplore.iee.org.

Digital Object Identifier 10.1109/TBME.2008.2003270 and walk to elderly adults who may require the assistance of a cane or a walker. Postural control consists of both postural steadiness associated with the ability to maintain balance during quiet standing and postural stability that is associated with the response to applied external stimuli and volitional postural movements [1]. The postural control system makes use of information from the visual, vestibular, and somatosensory systems [2]. There are many factors that potentially affect the postural control system and may lead to an increased risk of falling. These include health or medical conditions such as diabetes, peripheral neuropathy (PN), stroke, multiple sclerosis, Parkinson's disease, cerebral palsy, and obesity [3]-[5], [15], [23], [28], [29]. One of the most important determinants for the risk of falling is age [1], [6], [7], [10], [17]. As humans age, they experience reduced tactile and joint position sensitivity and increased reaction time [8], as well as reduced muscle mass [4].

Balance is achieved when the subject's center of gravity (COG) remains within the base of support. COG is the vertical projection of the center of mass onto the base of support. It is a whole body characteristic that is difficult to measure directly, so typically the center of pressure (COP) is used instead. COP is the location of the vertical ground reaction force on the surface upon which the subject stands. COP movements are used to control the horizontal displacements of the center of mass. In general, the COP varies about the COG, but with higher amplitude and higher frequency content [25]. Using a single force plate, it is the net COP from both feet that is measured [9]. Over an extended period of time of quiet stance, the average of the COP must equal the average of the COG [26].

During quiet standing, humans invariably sway to maintain balance, and this motion is measured using the anteriorposterior (AP) and the medial-lateral (ML) components of the net COP. Different control mechanisms and different muscle groups are used to control AP and ML motion [25]. There are numerous metrics or features of quiet standing sway that have been measured and statistically analyzed including time-based, time- and frequency-based (hybrid), and frequency-based characteristics [7], [10], [11]. The relative sensitivity, variation, and correlation of 13 groups of features was investigated in [1], and this list of features was meant to be representative rather than exhaustive. More recently, the reliability of $36 \mathrm{COP}$ features was analyzed in [12]. Differences in the quiet standing sway of healthy and disabled individuals have been reported using a variety of sway metrics [5], [18], [23], [28], [29]. With so many quiet standing sway features under consideration, there is 
no clear consensus as to which single metric or subset is most appropriate for describing the steadiness of young, old, healthy, and unhealthy individuals.

This paper proposes a simple way to combine a set of quiet standing postural sway features into a single composite feature $C$ that accounts for variation with age $a$. The features are sorted based on the $r^{2}$-values of their linear regression models. A weighted sum of selected sway features is used to form the composite feature with optimal weighting coefficients obtained using principal component analysis (PCA). Once a composite feature is defined, a linear regression model $f(a)$ for healthy individuals can be created. The composite feature for an individual then can be compared with the healthy control (HC) model using a quiet standing index $C^{\prime}(a)=C / f(a)$. The quiet standing index takes on the value $C^{\prime}(a)=0$ when the subject is perfectly motionless (a theoretical state only approached asymptotically). If the subject has a composite feature that exactly matches the HC model, then $C^{\prime}(a)=1$. Values of $C^{\prime}(a)$ satisfying $C^{\prime}(a) \gg 1$ indicate excessive postural sway and correspond to reduced steadiness. A combined reliability and sensitivity performance metric (described in Section III) is used to determine the optimal number of features for the composite feature $C$. Using prediction interval bands, the composite feature and quiet standing index of individuals can be compared with the $\mathrm{HC}$ group. An increase in the quiet standing index of an individual over time may indicate that the subject is beginning to experience a reduction in steadiness. The quiet standing indexes of diabetic and nondiabetic subjects, and subjects with and without PN, were computed. For PN subjects and diabetic PN subjects (DPN), statistically significant increases were found in comparison with the HC group.

\section{METHOD}

\section{A. Subjects and Testing Procedures}

The initial set of subjects consisted of 108 adults, ranging in age from 19 through 77 years. Thirty eight subjects were diagnosed by their primary care physicians with early mild type II diabetes. Ten of these diabetic subjects (diabetic neurologically intact (DNI) group) had normal peripheral nerve conduction velocity (NCV) tests, and the remaining 28 diabetic subjects were seen to have PN (DPN group). Twenty two nondiabetic subjects were shown to have PN (PN group). Thirty nine subjects, who were nondiabetic and neurological intact, constituted the $\mathrm{HC}$ group. The remaining nine subjects were not classified, and not included in this study because their minimum nerve conduction velocities fell within $\pm 2 \%$ of the NCV thresholds for a finding of PN. The inclusion of a small gap provided a clear border between the PN and non-PN groups. The physical characteristics of the groups of subjects under investigation are summarized in Table I. The number of males and females is almost identical for the HC and DNI groups, but the PN and DPN groups have an increased number of males.

An area plot of the age distributions of the subjects in Table I is shown in Fig. 1, where it is clear that the three pathological groups tend to consist of older subjects. A majority of the subjects were recruited from the Veterans Administration (VA)
TABLE I

MEAN PHysicAl CHARACTERISTICS OF THE SUBJECTS

\begin{tabular}{||l|c|c|c|c||}
\hline Group & $\begin{array}{c}\text { Number } \\
\text { (Male+Female) }\end{array}$ & $\begin{array}{c}\text { Age } \\
(\text { year })\end{array}$ & $\begin{array}{c}\text { Ht. } \\
(\mathrm{m})\end{array}$ & $\begin{array}{c}\text { Wt. } \\
(\mathrm{kg})\end{array}$ \\
\hline \hline HC & $39(19+20)$ & $51.1 \pm 14.9$ & 1.65 & 78.8 \\
DNI & $10(5+5)$ & $59.7 \pm 7.7$ & 1.73 & 96.1 \\
PN & $22(17+5)$ & $56.5 \pm 9.7$ & 1.71 & 89.6 \\
DPN & $28(21+7)$ & $60.1 \pm 6.8$ & 1.72 & 96.3 \\
\hline Total & $99(62+37)$ & $55.7 \pm 11.8$ & 1.70 & 88.2 \\
\hline
\end{tabular}

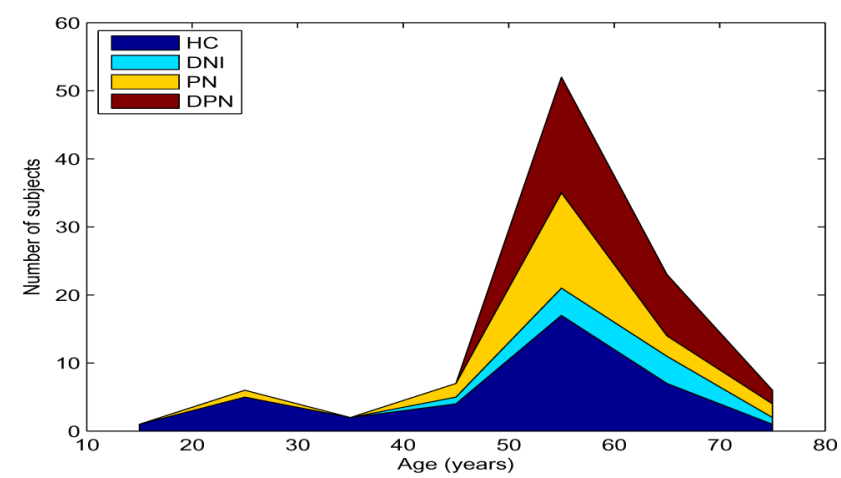

Fig. 1. Age distributions of the 39 subjects in the HC group, the 10 subjects in the DNI group, the 22 subjects in the PN group, and the 28 subjects in the DPN group. The values plotted at age 55 represent the number of subjects in the decade $50-60$.

Medical Centers (MCs), Shreveport, LA, and Pittsburgh, PA (Highland Drive). The remaining subjects were recruited from the community by advertising at Louisiana Tech University and in the Shreveport area. The recruiting, screening, testing, and informed consent procedures were reviewed and approved by the appropriate Institutional Review Boards.

All the subjects who were recruited for this investigation underwent visual, vestibular, auditory, musculoskeletal, and cognitive screening to maximize the liklihood that they had no undiagnosed conditions that may have affected their balance [13]. Subjects with respiratory dysfunction, cardiac condition, central nervous system disorder, musculoskeletal disorder, lower extremity amputation, severe arthritis, history of repeated falls, or who were currently taking medication to prevent dizziness were excluded.

The individuals with diabetes had mild type II diabetes as determined by their primary care physicians. All diabetic subjects had been diagnosed with diabetes within the past 10 years. They were using diet or oral medication to manage blood sugar levels, and they all reported stable blood sugar levels and acceptable hemoglobin A1C levels at the time of their testing.

Clinical nerve conduction tests on the lower extremities were performed by a VAMC neurology technician under the supervision of a neurologist. The norms used to classify subjects were taken from the Neurology Department at the Pittsburgh VAMC. NCV tests were performed on the tibeal, peroneal, and sural nerves of both legs, and the thresholds used were 41, 44, and $34 \mathrm{~m} / \mathrm{s}$, respectively. Each NCV was normalized by its threshold value, and the overall $\mathrm{NCV}$ score $X$ was set to the minimum of the normalized velocities. Subjects were classified as belonging to the PN group when $X \leq 0.98$, the NI group when 
$X \geq 1.02$, and the $\pm 2 \%$ gap when $0.98<X<1.02$. It was felt that including a gap would give a more reliable classification in comparison with classifying every subject as either NI or PN, even when the NCV score fell right on the boundary.

The experimental data were obtained using the SLIP-FALLS system, a sliding linear investigative platform for analyzing lower limb stability [14]. This is a computer-controlled airbearing mobile platform instrumented with a force plate to precisely measure COP [14]. For this study, the platform was held motionless, and the subjects stood barefoot with their arms at their sides. Throughout the data collection, the back of both heels were aligned in the frontal plane, with feet splayed out at natural stance. In the ML direction, the subjects were asked to maintain their normal width stance. In order to minimize the effects of visual and audio cues, the subjects were blindfolded, and headphones were used to provide masking noise (70-dB SPL) and instructions. The use of the eyes-closed condition during quiet stance did not appear to have any detrimental effects on the elderly or disabled subjects.

\section{B. Quiet Standing Features}

Quiet standing COP time-series measurements were taken for each subject. Here, $x_{\mathrm{AP}}(k)$ and $x_{\mathrm{ML}}(k)$ denote the $k$ th samples of the AP and ML components of the COP, respectively. The means of $x_{\mathrm{AP}}(k)$ and $x_{\mathrm{ML}}(k)$ have been removed as in [1]. Let $f_{s}$ denote the sampling frequency, and $N$ the total number of samples. For this investigation, the initial sampling frequency of $1000 \mathrm{~Hz}$ was higher than that needed for the COP measurements, so the time-series measurements were downsampled by a factor of 10 to yield $f_{s}=100 \mathrm{~Hz}$. There were three quiet standing trials, each of duration $20 \mathrm{~s}$, taken $30 \mathrm{~min}$ to $1 \mathrm{~h}$ apart. The first $5 \mathrm{~s}$ of each trial were removed to avoid potential startup transients [20]. After removing the means, the data segments were joined using cubic splines to yield a single segment consisting of $N=4500$ samples or $45 \mathrm{~s}$. This involved adjusting 4 points out of 4500 using cubic spline interpolation to avoid abrupt transitions at the segment boundaries. Since less than $0.09 \%$ of the points were adjusted to fit the segments together, this appeared to have negligible effects on the computed features. A more general procedure for fitting noisy segments of data together using cubic smoothing splines is described in [27]. By constructing one long segment, instead of averaging three shorter segments, the resolution of the power density spectrum was improved.

For convenience, a plot of $x_{\mathrm{AP}}$ versus $x_{\mathrm{ML}}$ will be referred to as a stabilogram [1], also called a statokinesigram [26]. There are numerous metrics or features that can be used to characterize a stabilogram. A list of the 11 features considered in this case is shown in Table II. The features in Table II are a subset of features defined in [1] and analyzed in [2]. All of the features in [1] start out by first removing the means of $x_{\mathrm{AP}}$ and $x_{\mathrm{ML}}$ so that only the variations from the means are analyzed. To reduce the number of equations in the following feature definitions, let $x$ denote $x_{\mathrm{AP}}$ or $x_{\mathrm{ML}}$, as appropriate. The first two distance measures characterizing sway displacement are as
TABLE II

Quiet Standing Postural Sway Features of 37 Healthy Adults, Ages 19 THROUGH 77

\begin{tabular}{||l|l|c|r|c||}
\hline \multicolumn{1}{|c|}{ Feature } & Type & $M_{k}$ & \multicolumn{1}{c||}{$r_{k}$} & Rank \\
\hline \hline mean_dist_AP & distance & $1.83 \times 10^{1}$ & 0.224 & 2 \\
mean_dist_ML & & $1.61 \times 10^{1}$ & -0.026 & 11 \\
rms_dist_AP & & $2.37 \times 10^{1}$ & 0.207 & 3 \\
rms_dist_ML & & $2.15 \times 10^{1}$ & 0.068 & 9 \\
mean_vel_AP & \multirow{2}{*}{ velocity } & $6.27 \times 10^{2}$ & 0.056 & 10 \\
mean_vel_ML & & $5.14 \times 10^{2}$ & 0.200 & 4 \\
area_cc & area & $1.43 \times 10^{4}$ & 0.173 & 5 \\
area_ce & & $6.53 \times 10^{3}$ & 0.131 & 6 \\
area_sway & & $2.97 \times 10^{3}$ & 0.121 & 7 \\
ave_power_AP & power & $5.62 \times 10^{2}$ & 0.225 & 1 \\
ave_power_ML & & $4.62 \times 10^{2}$ & 0.120 & 8 \\
\hline
\end{tabular}

For each feature, $M_{k}$ is the maximum value, $r_{k}$ is $r$-value of the linear regression model, and rank is the ranking based on $r_{k}^{2}$.

follows:

$$
\begin{aligned}
\text { mean_dist_X } & =\frac{1}{N} \sum_{k=1}^{N}|x(k)| \\
\text { rms_dist_X } & =\left[\frac{1}{N} \sum_{k=1}^{N} x^{2}(k)\right]^{1 / 2} .
\end{aligned}
$$

The mean velocity characterizes the average speed of the sway and is computed by dividing the total distance traveled by the duration of the experimental run. Three area measures have been proposed to approximate the area of the stabilogram. One measure, area_cc, represents the area of the $95 \%$ confidence circle, a circle that is expected to enclose approximately $95 \%$ of the points on the stabilogram path. Another measure, area_ce, is somewhat more general in that it uses the $95 \%$ bivariate confidence ellipse. Computation of these two area measures is described in [1]. A third area measure estimates the area enclosed per unit time. It is constructed by summing the areas of the triangles formed by successive pairs of points on the sway path, using the sway centroid as the third vertex [16].

The power measures of postural sway are based on the power density spectrum $S_{x}(i)=|X(i)|^{2} / N$, where $X(i)=$ $\operatorname{DFT}\{x(k)\}$ is the discrete Fourier transform. The average power is the average of the power density spectrum

$$
\text { ave_power_X }=\frac{1}{N} \sum_{i=1}^{N} S_{x}(i) \text {. }
$$

Each of the four groups of subjects in Table I was tested for outliers using the means of the features in Table II. Subjects who averaged more than 3.5 standard deviations from the mean were classified as outliers and removed. This resulted in two HC subjects, with mean deviations of +3.6 and +4.0 , being removed. There were no outliers among the other three groups.

The set of features in Table II was selected using the following criteria. First, the feature value should go to zero when the subject is perfectly motionless (a theoretical state only approached asymptotically). Second, the feature value should increase when the size of the stabilogram increases. As a stabilogram grows in size, the COP trajectory approaches the boundary of the base of support. Finally, the feature value should depend on all of 
TABLE III

Features Used for Composite Feature $C$

\begin{tabular}{||l|l|l|l||}
\hline \multicolumn{1}{|c|}{ Name } & \multicolumn{1}{c|}{$M_{k}$} & \multicolumn{1}{c||}{$r_{k}$} & \multicolumn{1}{c||}{$w_{k}$} \\
\hline \hline ave_power_AP & $5.62 \times 10^{2}$ & 0.2248 & 0.00105 \\
mean_dist_AP & $1.83 \times 10^{1}$ & 0.2241 & 0.0287 \\
rms_dist_AP & $2.37 \times 10^{1}$ & 0.2070 & 0.0257 \\
\hline
\end{tabular}

$M_{k}$ is the maximum value used for normalization, $r_{k}$ is the $r$-value of the linear regression model, and $w_{k}$ is the composite feature weight.

the points in the stabilogram path in the sense that varying any point should vary the value. The first criterion eliminates fractal dimension and principal angle features because they are not well defined when the stabilogram is reduced to a point. The second criterion eliminates features based on the shape of the power density spectrum because increasing the size of the stabilogram does not change, for example, the frequency centroid or the frequency dispersion. The third criterion eliminates range features because only the extreme points contribute to the range. It is also possible to have vector forms of the features listed in Table II, such as the mean vector distance and the rms vector distance. These features were eliminated based on redundancy arguments because AP and ML are the components of the position vector. By restricting the features under consideration to the features listed in Table II, the quiet standing index has a very simple physical interpretation that is easily understood and applied.

\section{Feature Models}

Table II was constructed using the HC group. Each feature was normalized by its maximum value $M_{k}$, and then a linear regression model was constructed by regarding the feature to be a function of the subject's age $a$. Here, $m_{k}$ and $b_{k}$ are the slope and intercept, respectively, for feature $k$.

$$
f_{k}(a)=m_{k} a+b_{k} .
$$

The $r_{k}$-values in Table II are the $r$-values of the linear regression models, where $r_{k}^{2}$ denotes the fraction of the total variance that is accounted for by the linear regression model, and the sign of $r_{k}$ is the sign of $m_{k}$. The Rank column in Table II is obtained by sorting the features based on decreasing values of $r_{k}^{2}$. The fact that the $r_{k}$-values are quite small is an indication that the slopes are very small in comparison with the variance present in the data. A positive $r_{k}$ indicates that the feature tends to increase with age. The results in Table II appear to be consistent with those reported in [1], where a comparison of 20 healthy young adults with 20 healthy elderly adults showed that, for all features for which the two groups showed significant differences, the mean value increased for the older group. The candidate features in Table II will eventually be reduced to the top three features listed in Table III. The justification for selecting three features is based on a combined reliability and sensitivity performance metric that is described in Section III.

\section{Composite Feature}

The selected features in Table III can be combined into a single composite feature. Let $g_{k}$ denote the $k$ th selected feature for $1 \leq k \leq q$. A composite feature is obtained by forming the

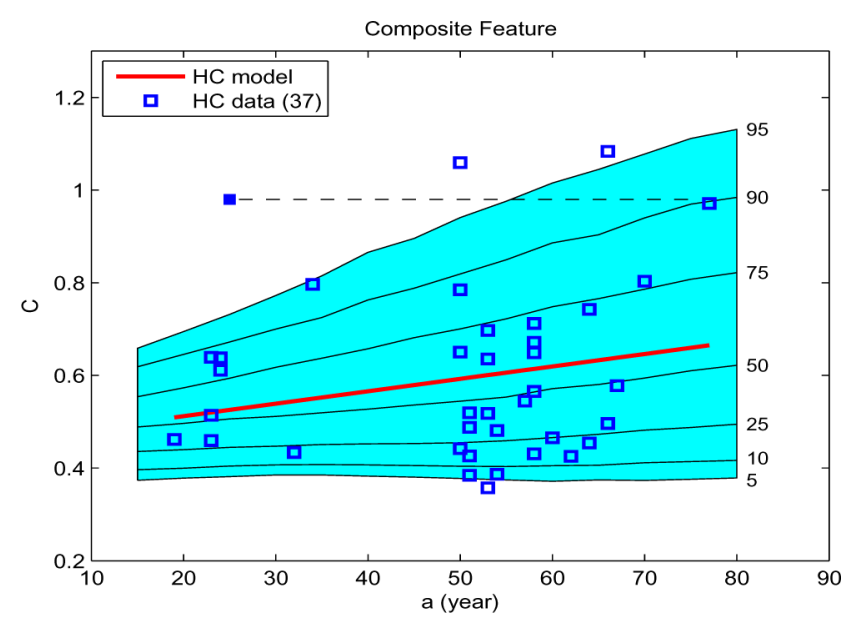

Fig. 2. Composite feature $C$ versus the age $a$ for the subjects in the HC group. The solid line is the linear regression HC model $f(a)$. The shaded region represents the 5\%-95\% prediction intervals for the composite features of the HC Group. The solid marker is moved left for illustration purposes.

following weighted average of $q$ features:

$$
C=\sum_{k=1}^{q} w_{k} g_{k} .
$$

The weighting coefficients are determined using a two-step process. First, each feature $g_{k}$ is normalized by dividing by its maximum value $M_{k}$. Normalization is useful because otherwise the composite value $C$ can be more sensitive to features with large means and variances. Next, a $q \times 1$ weight vector $v$ is computed using PCA [24]. The weight vector $w$ in (5) is then computed as $w_{k}=v_{k} / M_{k}$ for $1 \leq k \leq q$. The composite feature weights $w_{k}$ for the top three features are summarized in Table III. Since PCA weights are used in (5), the composite feature is the first principal component of the data generated by the features in Table III. The composite feature $C$ accounts for $96.9 \%$ of the total variance of the data using the features in Table III.

By using a single composite feature, one can develop a test for steadiness. To see this, let $a_{i}$ and $C_{i}$ denote the age and composite feature, respectively, for subject $i$. Next, let $f(a)$ be the linear regression model that fits the composite feature data $\left(a_{i}, C_{i}\right)$ for $1 \leq i \leq n$

$$
f(a)=m a+b .
$$

The composite features $C_{i}$ and linear regression model $f(a)$ for the HC group in Table I are shown in Fig. 2. Also shown are prediction intervals ${ }^{1}$ for the $\mathrm{HC}$ data computed using a bootstrap method, modified to account for linearly increasing standard deviation of the residuals [19]. The shaded area represents the area between the 5th and the 95th percentile. The heavy solid line is the composite feature linear regression model for the HC

\footnotetext{
${ }^{1}$ Whereas confidence intervals predict a range for a parameter of the data, prediction intervals are meant to describe the dataset itself. A $95 \%$ prediction interval is such that one would expect that $95 \%$ of the data points will fall within that interval.
} 
group using the features in Table III

$$
f(a)=0.0027 a+0.4584
$$

The $r$-value for the composite feature model, $r=0.2214$, is relatively small because the slope is only $m=0.0027$. However, the value of $f(a)$ does increase by $35.3 \%$ as age ranges from 20 to 80 years. From the spread in the prediction interval bands, it is evident that there is considerable variation in the composite feature data plotted in Fig. 2. Also, note that as the subjects grow older, there appears to be more variation in the composite feature. The prediction intervals are important for properly interpreting the value of the composite feature. To illustrate this (using an extreme case), the data point for a 77-year-old subject with a composite feature of $C=0.98$ was shifted left and replotted as a solid marker associated with a 25 -year-old. Clearly, the value of $C=0.98$ lies far above the 95th percentile when it is attached to a younger individual, but it is at the 90th percentile when evaluated at $a=77$ years.

As explained in this section (and amplified in Section IV), we have endeavored to show the thought process behind our definition of a composite feature. Although the weights shown in Table III might be altered by choosing a different (and/or larger) sample set, we are confident that these weights will provide a reasonable way to average the various measures of quiet standing postural sway. As such, we define the composite SLIP-FALLS sway statistic (CSSS), as the C-value computed using these weights, with composite SLIP-FALLS sway model $f(a)$. If other researchers are interested in doing an "apples to apples" comparison with their own subjects, they should use the weights in Table III.

\section{E. Quiet Standing Index}

Given the HC model, the quiet standing characteristics of subjects can be compared to one another in two ways. The most direct approach is to use the composite feature deviation

$$
\Delta C(a)=C-f(a) .
$$

The prediction interval bands give an indication of how far above or below the HC model a composite feature falls. An alternative approach is to normalize the composite feature by the $\mathrm{HC}$ model to produce the following quiet standing index:

$$
C^{\prime}(a)=\frac{C}{f(a)} .
$$

Two values of $C^{\prime}(a)$ have simple interpretations. The lower bound $C^{\prime}(a)=0$ occurs in the limit when the subject exhibits no sway whatsoever. If the composite feature of a subject exactly matches the $\mathrm{HC}$ model, then $C^{\prime}(a)=1$. A plot of the quiet standing indexes for the HC group is shown in Fig. 3. The mean of the quiet standing index, by construction, is $\mu=1$ and the standard deviation is $\sigma=0.29$.

For the quiet standing index, values of $C^{\prime}(a)$ satisfying $C^{\prime}(a) \gg 1$ indicate excessive sway. When the amplitude of the sway is sufficiently large, the COP of the subject begins to approach the boundary of the base of support. Consequently, very

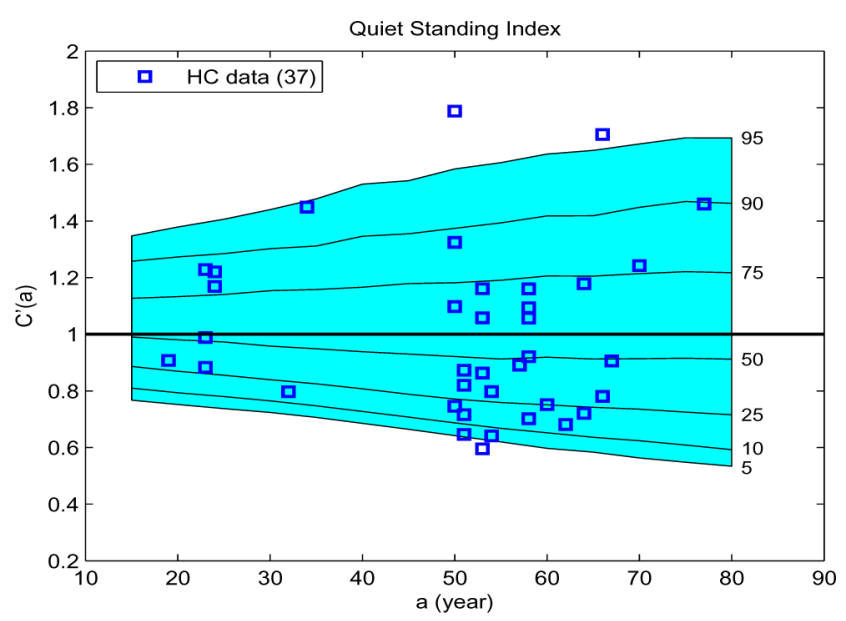

Fig. 3. Quiet standing index $C^{\prime}(a)$ versus age $a$ for the subjects in the HC group. The solid horizontal line corresponds to the linear regression $\mathrm{HC}$ model. The shaded region represents the $5 \%-95 \%$ prediction intervals for the quiet standing indexes of the $\mathrm{HC}$ group.

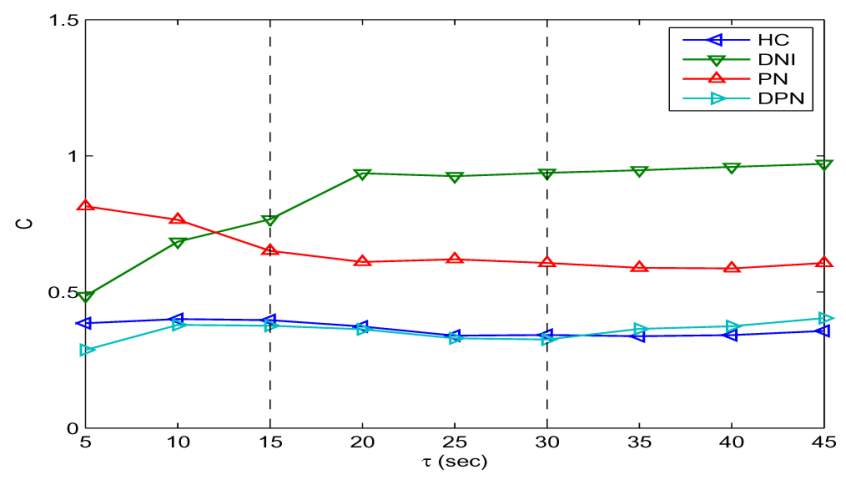

Fig. 4. Composite features versus the data duration $\tau$ using random subjects from the HC, DNI, PN, and DPN groups.

large values of the quiet standing index can be associated with a reduction in steadiness.

\section{RESUlts}

\section{A. Data Duration}

The effects of the quiet standing data duration on the value and the reliability of several individual postural sway features have been investigated [12], [21], [22]. These studies used data durations ranging from 10 to $120 \mathrm{~s}$ with up to eight trials conducted either consecutively or over two days. The reported results for the optimal number and length of epochs ranged from one epoch of 20-30 s [21] to up to seven epochs of $60 \mathrm{~s}$ depending on the particular sway feature used [12]. In view of these findings, it is useful to examine how sensitive the composite feature is to the duration of the data used to compute it. Fig. 4 shows a plot of the composite features of four subjects, selected at random from each group, as a function of the duration $\tau$ of the data used to compute $C$. The dotted vertical lines show the boundaries between the three epochs. For very short durations $(\tau \leq 20)$, there can be considerable fluctuation in the $C$-values. However, for longer durations $(\tau \geq 25)$, the composite features begin to stabilize and are approximately flat. Consequently, to measure 


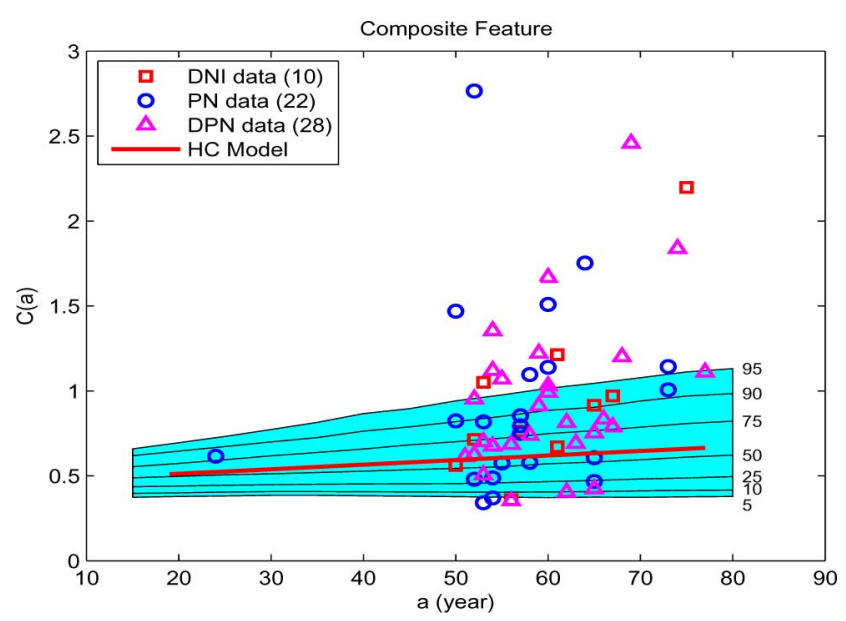

Fig. 5. Composite feature $C$ versus age $a$ for the 10 subjects in the diabetic (DNI) group, the 22 subjects in the PN group, and the 28 subjects in the DPN group. The shaded regions represent the 5\%-95\% prediction intervals for the composite features of the $\mathrm{HC}$ group.

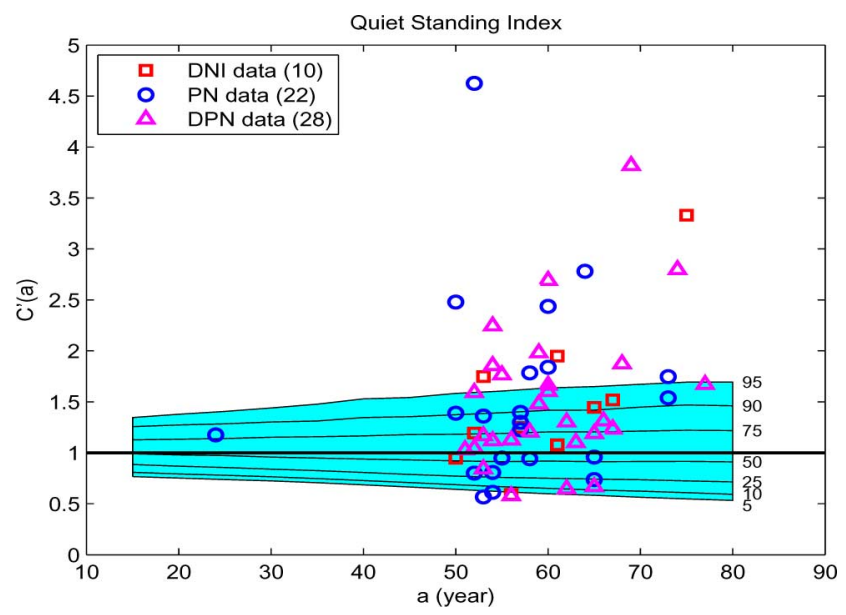

Fig. 6. Quiet standing index $C^{\prime}$ versus age $a$ for the 10 subjects in the diabetic (DNI) group, the 22 subjects in the PN group, and the 28 subjects in the DPN group. The shaded regions represent the 5\%-95\% prediction intervals for the quiet standing indexes of the $\mathrm{HC}$ group.

the composite feature, at least two epochs should be used. The fact that two to three epochs appear to suffice is perhaps a consequence of the fact that the composite feature $C$ is based on a weighted average of several normalized features rather than on a single feature.

\section{B. Subjects With Diabetes and PN}

Next, it is of interest to examine the composite features and quiet standing indexes of subjects who have been diagnosed with health conditions such as diabetes and/or PN. The second group in Table I consists of DNI subjects. The composite features and the quiet standing indexes of the ten DNI subjects are shown in Figs. 5 and 6, respectively. In this case, a few of the subjects exhibit an elevated quite standing index, but the results are roughly similar to those of the HC group. The mean quiet standing index for the DNI group was $\mu=1.51$ and the standard deviation was $\sigma=0.75$. Three subjects out of ten fell above the

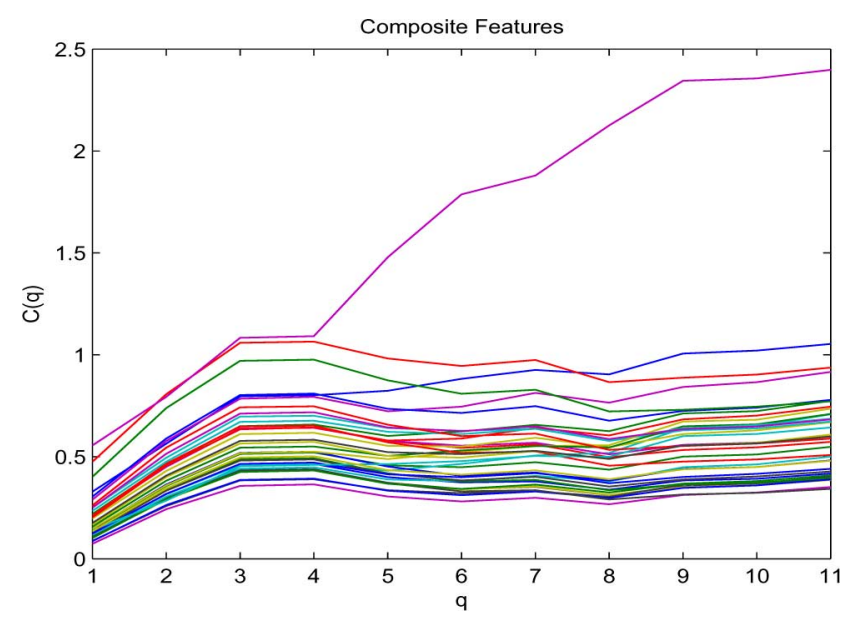

Fig. 7. Composite feature $C$ versus the number of component features $q$ for the 37 subjects in the $\mathrm{HC}$ group. The comb-like structure appearing in the interval $2 \leq q \leq 4$ represents a region where the relative ranking of subjects based on $C$ does not change. For $q>4$, an outlier type subject is present.

95th percentile band of the $\mathrm{HC}$ group, with one subject well above with a quiet standing index of $C^{\prime}(a)=3.33$.

The third group of subjects in Table I includes individuals who have PN but are not diabetic. The composite features and the quiet standing indexes of the $22 \mathrm{PN}$ subjects are also shown in Figs. 5 and 6, respectively. Of the 22 PN subjects, 7 exhibited quiet standing indexes above the 95th percentile of the $\mathrm{HC}$ group with the largest being $C^{\prime}(a)=4.62$. The mean quiet standing index for this group was $\mu=1.52$ and the standard deviation was $\sigma=0.92$. It is evident that there is somewhat more variation in $C^{\prime}(a)$ within the PN group.

The fourth group of subjects in Table I consists of individuals who are both diabetic and have PN (DPN). The composite features and the quiet standing indexes of the 28 DPN subjects are again shown in Figs. 5 and 6, respectively. Of the 28 subjects, 9 had quiet standing indexes above the 95th percentile of the HC group with the largest being $C^{\prime}(a)=3.82$. For the DPN subjects, the mean of the quiet standing index was $\mu=1.52$ and the standard deviation was $\sigma=0.71$.

\section{Feature Set Selection}

The feature set used for the composite feature $C$ was arrived at using a two-step procedure. First, the features were sorted according to decreasing $r^{2}$-values of their linear regression models. In this way, the effects of aging were taken into account. Next, the number of features $q$ was chosen to ensure that the composite feature $C$ is both reliable in terms of adding and subtracting features, and sensitive in terms of being able to distinguish between groups with different health characteristics. Let $C(i, q)$ denote the composite feature of subject $i$ using the first $q$ features from the sorted list. As the number of features $q$ increases, the ranking or relative position of subjects based on their $C$-values should not change or at least should exhibit minimal change. A range of values for $q$ over which the change appears to be minimal can be determined by plotting the composite features $C(i, q)$ for the subjects in the $\mathrm{HC}$ group, as shown in Fig. 7. A careful viewing of this dense family of 
curves reveals a comb-like structure in the range $2 \leq q \leq 4$. The appearance of parallel lines means that for these subjects, the values of $C$ relative to one another do not change. Although Fig. 7 contains considerable information, it can be difficult to analyze visually. The information in Fig. 7 can be distilled to a single variable by examining how the ordering of the subjects changes with $q$. Since there are $n$ subjects and $h$ features, $C$ is an $n \times h$ matrix. Let $[D, d]=\operatorname{sort}(C)$ be a sorted version of $C$ using the MATLAB sort function. Here, each column of $D$ contains the corresponding column of $C$, sorted according to increasing values of the composite feature. The $q$ th column of the matrix $d$ contains integers in the range $1-n$, which shows the ordering of the subjects based on their composite features when $q$ features are used. In order to determine an optimal value for $q$, consider how the ordering of the subjects changes as the number of features changes. A significant change in the ordering of the subjects when a single feature is added or removed would suggest that the composite feature might not be reliable because its value would be highly sensitive to the addition or removal of a single feature. Let $i(q)$ be the number of elements in the $q$ th column of $d$ that change as the number of features is increased from $q$ to $q+1$. Then, the percentage of subjects whose ranking does not change is as follows, where $n$ is the number of subjects:

$$
I(q)=100\left[1-\frac{i(q)}{n}\right] .
$$

Note that $I(q)$ ranges from $0 \%$ to $100 \%$, with 100 corresponding to the case when the ordering of the subjects based on $C$ does not change as the number of features is increased from $q$ to $q+1$. The measure in (10) represents the reliability of $C$ over the interval $[q, q+1]$. In order to develop a reliability measure corresponding to the number of features $q$, one can take the average of $I(q-1)$ and $I(q)$.

$$
R(q)=\frac{I(q-1)+I(q)}{2} \%, \quad 1<q<h .
$$

Note that $R(q)$ takes into account the effects of either adding or removing a feature from $C$. At the end point $q=1$, no features can be removed, so $R(1)=I(1)$, and when $q=h$, no features can be added, so $R(h)=I(h)$.

It is also useful to develop a quiet standing index that is sensitive in the sense that it is able to distinguish between different groups of individuals who may have health conditions that affect their balance. A one-way analysis of variance (ANOVA) was performed on the four groups. Given the null hypothesis that there is no statistically significant difference between the means of the groups, the computed $p$-value specifies the probability that the null hypothesis is true. Let $P(q)$ be the $p$-value using $q$ features to form the quiet standing index. Then, the number of features at which the quiet standing index is most sensitive corresponds to the minimum value of $P(q)$.

The two characteristics, reliability and sensitivity, can be combined into a single performance metric that depends on the number of features $q$

$$
V(q)=\left(\frac{P_{\min }}{R_{\max }}\right) \frac{R(q)}{P(q)}, \quad 1 \leq q \leq h .
$$

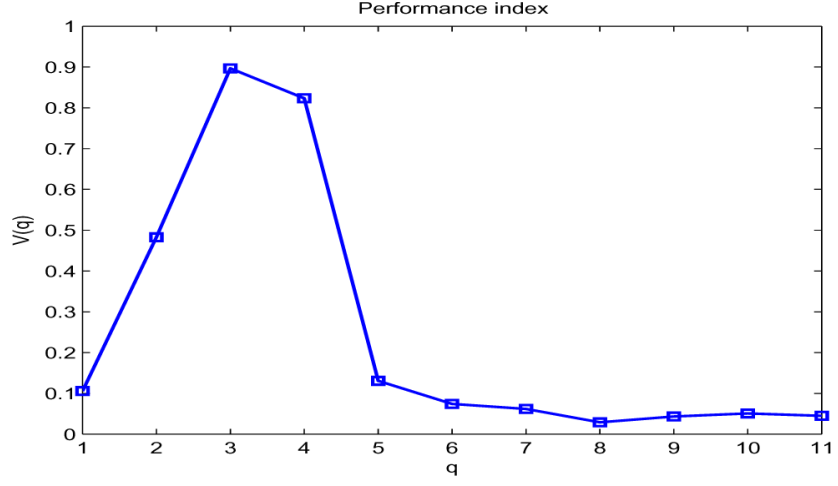

Fig. 8. Performance index $V(q)$ that takes into account the reliability of adding or subtracting features and the sensitivity in distinguishing between different groups of subjects.

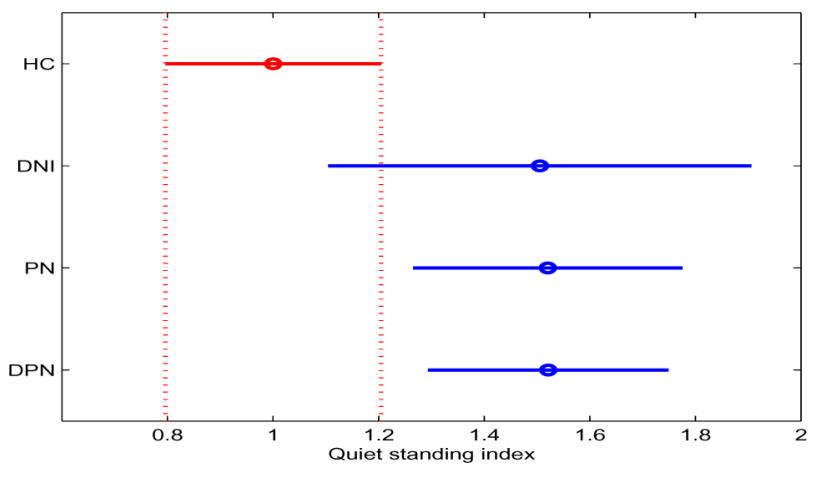

Fig. 9. Multiple comparison of the quiet standing indexes of the four groups of subjects. There are statistically significant increases in the means of the PN and DPN groups in comparison with the $\mathrm{HC}$ group since the $95 \%$ confidence intervals do not overlap.

Here, $R_{\max }=70.3 \%$ is the maximum value achieved by the reliability measure, while $P_{\min }=0.0038$ is the minimum value achieved by the sensitivity measure. If $R(q)$ and $P(q)$ were to achieve their extrema at the same number of features, then the peak value of the combined performance metric $V(q)$ would be one. A plot of the performance metric $V(q)$ is shown in Fig. 8. There is a peak at $V(3)=0.90$, thus confirming how the three features listed in Table III were chosen. Observe from Fig. 8 that when three features are used, the performance is 8.5 times higher than the performance obtained using a single feature.

\section{Ability to Discriminate}

A one-way ANOVA of the quiet standing indexes of the four groups was performed in order to test the ability of $C^{\prime}$ to discriminate between the groups. It should be noted (see Fig. 1) that the HC group contains more young subjects than the other groups. The results indicated that the quiet standing index did detect a statistically significant difference between the four groups of subjects with a $p$-value of $p=0.0038$. A multiple comparison was then made to see how the means of the groups differed from one another with the results shown graphically in Fig. 9.

Both the PN subjects and the DPN subjects showed statistically significant increases in the quiet standing index in comparison with the $\mathrm{HC}$ group where it can be seen in Fig. 9 that the 
TABLE IV

QuiET STANDING INDEX CHARACTERISTICS OF THE GROUPS OF SUBJECTS

\begin{tabular}{||l|c|c|c|r||}
\hline Group & $n$ & $\mu$ & $\sigma$ & $C^{\prime}>95 \%$ \\
\hline \hline HC & 37 & 1.00 & 0.29 & $5.6 \%$ \\
DNI & 10 & 1.51 & 0.75 & $30.0 \%$ \\
PN & 22 & 1.52 & 0.92 & $31.8 \%$ \\
DPN & 28 & 1.52 & 0.71 & $32.1 \%$ \\
\hline
\end{tabular}

Here, $n$ is the number of subjects, $\mu$ is the mean, $\sigma$ is the standar deviation, and $c^{\prime}>95 \%$ is the percent of subjects that lie above the $95^{\text {th }}$ percentile of the $\mathrm{HC}$ group.

95\% confidence intervals do not overlap. For the DNI subjects, the increase in the mean of the quiet standing index was not statistically significant. The absence of a statistically significant difference between the DNI and HC groups is consistent with results reported in [29]. The fact that PN and DPN subjects appeared less steady makes sense in terms of the underlying postural control system. Low nerve conduction velocities imply more delay in the feedback control system used to maintain balance. It is well known that as delay is increased in a linear feedback control system, the stability margin decreases.

A summary of the quiet standing index characteristics of the four groups is shown in Table IV. For each group, the increase in the mean quiet standing index was influenced by individuals whose quiet standing indexes fell above the 95th percentile of the HC group. The last column in Table IV shows the percentage of subjects within each group whose $C^{\prime}$-values were above the 95th percentile of the HC group.

\section{DISCUSSION}

There are several novel characteristics that set the composite feature and quiet standing index apart from other postural sway metrics that have been proposed. The first is the technique used to sort the individual sway features based on their sensitivity to age using the $r^{2}$-values of their linear regression models. An alternative way to select features was introduced in [22] and applied in [5]. Here, 14 postural sway features commonly used in clinical practice were analyzed using PCA. For 19 Parkinson's disease subjects in the levodopa off state, it was found that the first four principal components (weighted sums of the 14 features) were sufficient to account for $94.7 \%$ of the total variation in the data. The individual sway features with the most significant contributions to each of the principal components were then identified using a process described in [22]. A similar analysis was applied to the Parkinson's disease subjects in the levodopa on state where it was found that the first three principal components (different from the off state) account for $93.0 \%$ of the total variation in the data. Using this technique, significant groups of sway features were identified, and individual features were ordered or ranked within each group. This is in contrast to the proposed approach where all features are initially ranked with respect to their sensitivity to age using the $r^{2}$-values of their linear regression models.

The second unique characteristic of the composite feature $C$ is the determination of an optimal number of individual sway features. For each fixed number of features $q$ for $1 \leq q \leq h$, a PCA was used to compute optimal weights. The composite feature $C$ corresponds to the first principal component and it accounts for the maximum variance in the data. For each $q$, the subjects were sorted according to increasing values of $C$. To determine the optimal number of features, the sensitivity of the ordering to changes in the number of features was computed. This led to a reliability measure $R(q)$ that specifies the percentage of subjects whose position in the sorted list does not change when a feature is added or removed. Thus, $R(q)$ represents the reliability of using $C$ to rank the subjects. The sensitivity of the quiet standing index $C^{\prime}(a)$ in being able to distinguish between groups of subjects with different health characteristics was also examined by computing the $p$-value $P(q)$ using a oneway ANOVA. These two characteristics, reliability and sensitivity, were then combined into a single performance metric $V(q)$ whose peak value was used to determine the optimal number of features. The optimum of $q=3$ features achieved a reliability of $\mathrm{R}(3)=70.3 \%$ and a sensitivity of $P(3)=0.0038$. The number of individual features in [5] was fixed at $q=14$ and the number of principal components was allowed to vary until at least $90 \%$ of the variance was accounted for. For the composite feature $C$, the number of principal components is fixed at one, but the number of individual features that contribute to it was allowed to vary, and it was found that the optimal number from the sorted list was $q=3$. The composite feature $C$ accounts for $96.9 \%$ of the total variance in the data associated with the selected features.

A third novel characteristic of the quiet standing index is that it explicitly incorporates the effects of aging. Using the composite feature $C$, a linear regression model was developed for $\mathrm{HC}$ subjects that shows how the composite feature varies with age. Normalization of the composite feature by the $\mathrm{HC}$ model then yields the quiet standing index $C^{\prime}(a)$, a postural sway metric that takes the age of the subject into account. Prediction interval bands that show the percentiles of HC subjects who are expected to have $C$ - and $C^{\prime}$-values below a given threshold were also computed. These prediction interval bands can be used in a fashion similar to the clinical growth charts that physicians use to chart the development of children as their height and weight change with age as they mature.

The three features that contribute to the composite feature $C$ include one power feature (ave_power_AP) and two distance features (mean_dist_AP and rms_dist_AP). The average power feature uses the information contained in the power density spectrum, and it does so with a metric that goes to zero when the subject is motionless and increases in value as the size of the stabilogram increases. Using both mean distance and rms distance provides for applying different importance values for outlier type motion. Note that the performance in Fig. 8 is almost is high when four features are used. Including the fourth feature, mean_vel_ML, adds not only another type of feature (velocity) but also another direction (ML). However, when four features are used, the PCA weights are $v=[0.592,0.526,0.609,0.042]$. Here, the fourth weight is more than a full order of magnitude smaller than the other weights, and this suggests that mean_vel_ML does not contribute significantly to $C$. The fifth feature in the sorted list is area_cc, and with it, all four groups of features from Table II would be represented. However, it is 
clear from Fig. 8 that when five features are used to compute $C$, the performance decreases to a value of $V(5)=0.131$ that is only marginally better than the $V(1)=0.106$ achieved using a single feature. When three features are used, the performance is 8.5 times higher than the performance obtained using a single feature.

In most of the studies of postural sway, analysis of quiet standing for an individual has been viewed in relation to a larger sample group. In a clinical setting, there is only one samplethe person being tested. Interpretation of the COP measurement requires an understanding of the "normal range," where a number of studies indicate that this normal should vary with age. In particular, we note that a sway value that might be "healthy" for an elderly patient could be viewed as "excessive" for a young adult (e.g., see the dashed line in Fig. 2). Similarly, our model attempts to distinguish between the normal growth of sway due to aging and the excessive growth of sway that might indicate a true postural instability. Our methodology provides a means for evaluation of these single sample points without requiring a direct comparison group.

The composite feature and the quiet standing index may be useful clinical tools to assess a balance dysfunction when working with individuals with balance disorders. The quiet standing index combines several different postural sway characteristics into a single scalar score $C^{\prime}$ that is easily understood by both clinicians and patients. Fig. 9 shows that this score is able to distinguish between adults with balance impairment and adults without balance deficits. The quiet standing index may also be more sensitive to change after rehabilitation than other measures. However, further research is necessary to evaluate that hypothesis. Applying the quiet standing index to other patient populations would also be an area for further research. As noted previously, we assert that the weighting coefficients in Table III and the model in (7) provide a reasoned way to express a composite feature, and researchers/clinicians interested in applying this technique should use these values in computing the CSSS. Note from Fig. 1 that most of the HC data came from subjects in the age range from 45 to 65 years. It is acknowledged that the $\mathrm{HC}$ model might benefit from additional data from young and middle aged subjects. Similarly the prediction intervals (which help to describe what is normal, marginal, or outlier) could also benefit from additional data on healthy individuals. Prediction intervals based on the bootstrap method for the composite feature $C$ and the quiet standing index $C^{\prime}$ are summarized in Tables V and VI, respectively [19]. We expect to refine the curves defined in Tables V and VI as more data become available.

One of the useful characteristics of the power and distance metrics used to form the composite feature is that they can be computed in real time. The features in Table III assume that the means of the COP data have been removed. The mean itself can be computed in real time using a recursive formulation. By using a real-time formulation of $C^{\prime}$, a subject can be evaluated using a single quiet standing trial, blind folded and lasting up to $60 \mathrm{~s}$ perhaps. The measured value of $C^{\prime}$ can be displayed, and it should stabilize once sufficient time has elapsed.

The examined subjects included DNI, PN, and DPN groups. In each case, the percentage of subjects with $C^{\prime}$-values above
TABLE V

Bootstrap PREDICTION INTERVALS FOR THE COMPOSITE FEATURE $C$ USING THE HC SUBJECTS

\begin{tabular}{||c|c|c|c|c|c|c|c||}
\hline \multirow{2}{*}{ Age } & \multicolumn{7}{|c||}{ Percentile } \\
\cline { 2 - 8 } & 5 & 10 & 25 & 50 & 75 & 90 & 95 \\
\hline \hline 15 & 0.380 & 0.402 & 0.442 & 0.494 & 0.559 & 0.620 & 0.660 \\
20 & 0.387 & 0.408 & 0.448 & 0.504 & 0.581 & 0.649 & 0.697 \\
25 & 0.390 & 0.411 & 0.451 & 0.510 & 0.602 & 0.680 & 0.740 \\
30 & 0.392 & 0.413 & 0.453 & 0.515 & 0.623 & 0.706 & 0.778 \\
35 & 0.390 & 0.412 & 0.453 & 0.520 & 0.645 & 0.735 & 0.823 \\
40 & 0.391 & 0.414 & 0.458 & 0.528 & 0.666 & 0.763 & 0.858 \\
45 & 0.388 & 0.413 & 0.459 & 0.536 & 0.690 & 0.797 & 0.906 \\
50 & 0.382 & 0.409 & 0.462 & 0.549 & 0.714 & 0.828 & 0.951 \\
55 & 0.382 & 0.409 & 0.464 & 0.556 & 0.737 & 0.860 & 0.988 \\
60 & 0.379 & 0.409 & 0.470 & 0.568 & 0.758 & 0.893 & 1.029 \\
65 & 0.373 & 0.408 & 0.474 & 0.576 & 0.773 & 0.921 & 1.062 \\
70 & 0.376 & 0.411 & 0.481 & 0.594 & 0.795 & 0.947 & 1.083 \\
75 & 0.376 & 0.413 & 0.487 & 0.608 & 0.814 & 0.974 & 1.123 \\
80 & 0.379 & 0.419 & 0.497 & 0.619 & 0.833 & 0.997 & 1.144 \\
\hline
\end{tabular}

TABLE VI

BOOTSTRAP PREDICTION INTERVALS FOR THE QUIET STANDING INDEX $C^{\prime}$ USING THE HC SUBJECTS

\begin{tabular}{||c|c|c|c|c|c|c|c||}
\hline \multirow{2}{*}{ Age } & \multicolumn{7}{|c||}{ Percentile } \\
\cline { 2 - 8 } & 5 & 10 & 25 & 50 & 75 & 90 & 95 \\
\hline \hline 15 & 0.770 & 0.811 & 0.889 & 0.989 & 1.122 & 1.247 & 1.335 \\
20 & 0.761 & 0.800 & 0.876 & 0.983 & 1.136 & 1.267 & 1.366 \\
25 & 0.746 & 0.785 & 0.859 & 0.968 & 1.143 & 1.288 & 1.409 \\
30 & 0.729 & 0.768 & 0.840 & 0.953 & 1.152 & 1.301 & 1.437 \\
35 & 0.709 & 0.747 & 0.821 & 0.939 & 1.160 & 1.316 & 1.479 \\
40 & 0.691 & 0.733 & 0.809 & 0.931 & 1.170 & 1.332 & 1.501 \\
45 & 0.670 & 0.713 & 0.791 & 0.922 & 1.180 & 1.356 & 1.545 \\
50 & 0.644 & 0.688 & 0.776 & 0.920 & 1.193 & 1.377 & 1.586 \\
55 & 0.626 & 0.671 & 0.760 & 0.910 & 1.202 & 1.396 & 1.610 \\
60 & 0.604 & 0.653 & 0.750 & 0.908 & 1.210 & 1.420 & 1.644 \\
65 & 0.577 & 0.632 & 0.736 & 0.899 & 1.210 & 1.435 & 1.664 \\
70 & 0.562 & 0.619 & 0.726 & 0.905 & 1.219 & 1.449 & 1.668 \\
75 & 0.543 & 0.601 & 0.718 & 0.905 & 1.219 & 1.466 & 1.700 \\
80 & 0.528 & 0.590 & 0.712 & 0.901 & 1.224 & 1.475 & 1.701 \\
\hline
\end{tabular}

the 95th percentile band of the HC group was approximately six times that of the HC group. Using a one-way ANOVA, the quiet standing indexes of the PN and DPN groups showed statistically significant increases over the HC group. The smaller DNI group did not show a statistically significant increase at the 95\% confidence level, a finding that is consistent with results reported in [29]. Besides comparing groups with different physical characteristics, the quiet standing index also can be used to compare individual subjects within a group. Another potentially useful way to apply the quiet standing index is to monitor how $C^{\prime}$ varies for an individual over time. By measuring the quiet standing index of an individual every year or every few years, and computing a trend line, a potentially troublesome change in the quiet standing index might be detected. If the measured $C^{\prime}>1$ and the trend is sharply upward, then this may indicate the beginning of a reduction in steadiness and an increased risk of falling.

All the subjects considered here underwent lower limb clinical nerve conduction studies as part of the study protocol. Thus, their neurological status was known. Future papers will apply this technique to additional more than 90 subjects (many in the 18- to 35-year-old range) who did not have NCV tests done. We also plan to use additional (repeat) observations from many of the subjects in the present paper. An aggregate of 
approximately $6000 \mathrm{~s}$ of quiet standing data was considered here. An additional aggregate of $15000 \mathrm{~s}$ of data is being analyzed to further validate this approach.

\section{ACKNOWLEDGMENT}

The authors would like to thank X. Dong, R. B. Pilkar, V. B. Bhatkar, and past members of the SLIP-FALLS group for their help. They thank all of the Ph.D., M.S., and B.S. students in the SLIP-FALLS laboratory who helped in data collection over a number of years.

\section{REFERENCES}

[1] T. E. Prieto, J. B. Myklebust, R. G. Hoffmann, E. G. Lovett, and B. M. Myklebust, "Measures of postural steadiness: Differences between healthy young and elderly adults," IEEE Trans. Biomed. Eng., vol. 43, no. 9, pp. 956-966, Sep. 1996.

[2] H. C. Diener and J. Dichgans, "On the role of vestibular, visual, and somatosensory information for dynamic postural control in humans," in Progress in Brain Research, O. Pompeiano and J. H. J. Allum, Eds. Amsterdam: Elsevier, 1988, vol. 76, pp. 253-262.

[3] W. Owusu, W. Willet, A. Ascherio, D. Spiegelman, E. Rimm, D. Feskanich, and G. Colditz, "Body anthropometry and the risk of hip and wrist fractures in men: Results from a prospective study," Obes. Res., vol. 5, pp. 12-19, 1998.

[4] P. Corbeil, M. Simoneau, D. Rancourt, A. Tremblay, and N. Teasdale, "Increased risk of falling associated with obesity: Mathematical modeling of postural control," IEEE Trans. Neural Syst. Rehabil. Eng., vol. 9, no. 2, pp. 126-136, Jun. 2001.

[5] L. Rocchi, L. Chiari, A. Cappello, and F. B. Horak, "Identification of distinct characteristics of postural sway in Parkinson's disease: A feature selection procedure based on principal component analysis," Neurosci. Lett., vol. 394, pp. 140-145, 2006.

[6] S. R. Cummings and M. C. Nevitt, "A hypothesis: The causes of hip fractures," J. Gerontol., vol. M-44, pp. 107-111, 1989.

[7] T. E. Prieto, J. B. Myklebust, and B. M. Myklebust, "Characterization and modeling of postural steadiness in the elderly: A review," IEEE Trans. Rehabil. Eng., vol. 1, no. 1, pp. 26-34, Mar. 1993.

[8] S. R. Lord, R. D. Clark, and I. W. Webster, "Postural stability and associated physiological factors in a population of aged persons," J. Gerontol., vol. 46, pp. 69-76, 1991.

[9] P. R. Rougier, "Relative contribution of the pressure variations under the feet and body weight distribution over both legs in the control of upright stance," J. Biomech., vol. 40, pp. 2477-2482, 2007.

[10] B. E. Maki, P. J. Holliday, and G. R. Fernie, "Aging and postural control: A comparison of spontaneous- and induced-sway balance tests," J. Amer. Geriatr. Soc., vol. 38, pp. 1-9, 1990.

[11] P. J. Loughlin and M. S. Redfern, "Spectral characteristics of visually induced postural sway in healthy elderly and healthy young subjects," IEEE Trans. Neural Syst. Rehabil. Eng., vol. 9, no. 1, pp. 24-30, Mar. 2001.

[12] B. R. Santos, A. Delisle, C. Lariviere, A. Plamondon, and D. Imbeau, "Reliability of centre of pressure summary measures of postural steadiness in healthy young adults," Gait Posture, vol. 27, no. 3, pp. 408-415, 2008.

[13] S. J. Richerson, S. M Morstatt, K. K. O Neil, G. Patrick, and C. J. Robinson, "Effect of lateral perturbations on psychophysical acceleration detection thresholds," J. Neuroeng. Rehabil., vol. 3, no. 2, 2006, doi:10.1186/17430003-3-2.

[14] C. J. Robinson, M. C. Purucker, and L. W. Faulkner, "Design, control, and characterization of a sliding linear investigative platform for analyzing lower limb stability (SLIP-FALLs)," IEEE Trans. Rehabil. Eng., vol. 6, no. 3, pp. 334-350, Sep. 1998.

[15] M. L. Corradini, "Early recognition of postural disorder in multiple sclerosis through movement analysis: A modeling study," IEEE Trans. Biomed. Eng., vol. 44, no. 11, pp. 1029-1038, Nov. 1997.

[16] A. Hurschmidt, J. Dichgans, K. H. Mauritz, and M. Hufschmidt, "Some methods and parameters of body sway quantification and their neurological applications," Arch. Psychiatr. Nervenkr., vol. 228, pp. 135-150, 1980.

[17] A. Nardone, M. Grasso, and M. Schieppati, "Balance control in peripheral neuropathy: Are patients equally unstable under statis and dynamic conditions?," Gait Posture, vol. 23, no. 3, pp. 364-373, 2006.
[18] H. C. Diener, J. Dichgans, M. Bacher, and B. Gompf, "Quantification of postural sway in normals and patients with cerebellar diseases," Electroencephalogr. Clin. Neurophysiol., vol. 57, pp. 134-142, 1984.

[19] J. P. Lam and M. R. Veall, "Bootstrap prediction intervals for single period regression forecasts," Int. J. Forecasting, vol. 18, no. 1, pp. 125-130, 2002.

[20] K. LeClair and C. Riach, "Postural stability measures: What to measure and for how long," Clin. Biomech., vol. 11, pp. 176-178, 1996.

[21] M. G. Carpenter, J. S. Frank, D. A. Winter, and G. W. Peysar, "Sampling duration effects on centre of pressure summary measures," Gait Posture, vol. 13, pp. 35-40, 2001.

[22] L. Rocchi, L. Chiari, and A. Cappello, "Feature selection of stabilometric parameters based on principal component analysis," Med. Biol. Eng. Comput., vol. 42, pp. 71-79, 2004.

[23] G. R. Harris, S. A. Riedel, D. Matesi, and P. Smith, "Standing postural stability assessment and signal stationarity in children with cerebral palsy," IEEE Trans. Rehabil. Eng., vol. 1, no. 1, pp. 33-42, Mar. 1993.

[24] I. T. Jolliffe, Principal Component Analysis. New York: SpringerVerlag, 1986.

[25] D. A. Winter, F. Prince, J. S. Frank, C. Powell, and K. F. Zabjek, "Unified theory regarding $\mathrm{A} / \mathrm{P}$ and $\mathrm{M} / \mathrm{L}$ balance in quiet standing," J. Neurophysiol., vol. 75, pp. 2334-2343, 1996.

[26] D. A. Winter, "Human balance and posture control during standing and walking," Gate Posture, vol. 3, no. 4, pp. 193-214, 1995.

[27] M. F. Hutchinson, "A fast procedure for calculating minimum crossvalidation cubic smoothing splines," ACM Trans. Math. Softw., vol. 12, no. 2, pp. 150-153, 1986.

[28] S. F. Donker, A. Ledebt, M. Roerdink, G. J. P. Savelsbergh, and P. J. Beek, "Children with cerebral palsy exhibit greater and more regular postural sway than typically developing children," J. Exp. Brain Res., vol. 184, pp. 363-370, 2008.

[29] G. G. Simoneau, J. S. Ulbrecht, J. A. Derr, M. B. Becker, and P. R. Cavanagh, "Postural instability in patients with diabetic sensory neuropathy," Diabetes Care, vol. 17, no. 12, pp. 1411-1421, 1994.

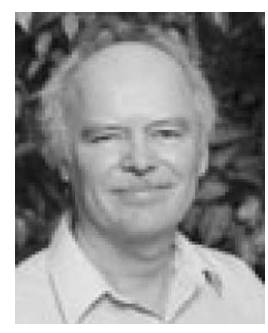

Robert J. Schilling (SM'89) received the B.E.E. degree from the University of Minnesota, Minneapolis, in 1969, and the M.S. and Ph.D. degrees from the University of California, Berkeley, in 1970 and 1973, respectively, all in electrical engineering.

From 1974 to 1978 , he was a Lecturer in the Department of Electrical Engineering and Computer Science, University of California, Santa Barbara. In 1978, he joined the Department of Electrical and Computer Engineering, Clarkson University, Potsdam, NY, where he is currently a Full Professor. He has authored or coauthored four textbooks in the areas of engineering analysis, robotics, numerical methods, and digital signal processing. His current research interests include adaptive signal processing, active noise control, and control and identification of nonlinear systems.

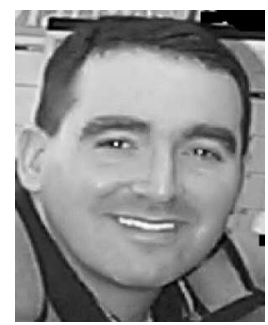

Erik M. Bollt received the B.A. degree from the University of California, Berkeley, in 1990, and the M.S. and Ph.D. degrees from the University of Colorado, Boulder, in 1992 and 1995, respectively, all in applied mathematics.

Since 2002, he has been a member of Mathematics and Computer Science Faculty, jointly with the Department of Physics, Clarkson University, Potsdam, NY, where he is currently a Full Professor. His current research research interests include dynamical systems, including fundamental issues of topological dynamics, symbolic dynamics, measurable dynamics, and transfer operators, as well as stochastic dynamical systems. Additionally, as an applied mathematician, he has worked on problems involving control theory applications including air foil flutter suppression, information processing, image object detection, control of chaos, physiological data processing, and modeling, as well as developing a deeper understanding of both dynamics of networks and dynamics on networks including applications in communications and social and epidemiological settings. 


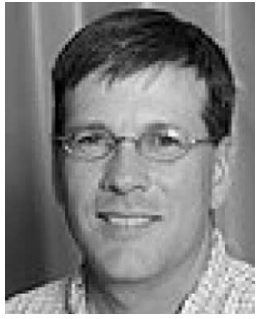

George D. Fulk received the B.A. degree in political science from Brandeis University, Waltham, MA, in 1986, the M.S. degree in physical therapy from the University of Massachusetts, Lowell, in 1994, and the $\mathrm{Ph} . \mathrm{D}$. degree in physical therapy from Nova Southeastern University, Ft. Lauderdale, FL, in 2005.

$\mathrm{He}$ is currently an Assistant Professor in the Department of Physical Therapy, Clarkson University, Potsdam, NY. His current research interests include the application of rehabilitation engineering technologies to enhance motor recovery and quality of life after neurological injury and in outcome measures designed to assess clinically meaningful change in people with neurological disorders.

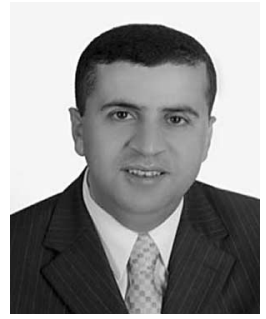

Ahmad F. Al-Ajlouni (M'97) received the M.S. degree in electrical engineering from Manhattan College, New York, in 1989, and the Ph.D. degree in electrical and computer engineering from Clarkson University, Potsdam, NY, in 1997.

$\mathrm{He}$ is currently an Associate professor in the Department of Communication Engineering, Hijjawi Faculty of Engineering Technology, Yarmouk University, Irbid, Jordan. His current research interests include the areas of data compression, digital sigidentification, and artificial intelligence. nal processing, active noise control, nonlinear system

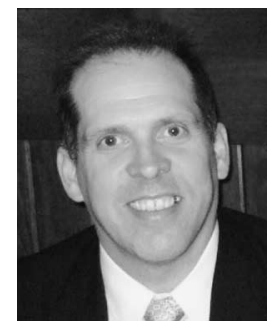

Joseph D. Skufca received the B.S. degree in English from the United States Naval Academy, Annapolis, MD, in 1985, and the M.S. and Ph.D. degrees in applied mathematics from the University of Maryland, College Park, in 2003 and 2005, respectively.

He served 20 years in the Submarine Force, with sea tours aboard both fast attack and ballistic missile submarine of both the Atlantic and Pacific Fleets. He was a Submarine Security Technology Requirements Officer as well as an Instructor and a Company Officer at the United States Naval Academy. He retired from active duty in 2005. In 2005, he joined the Faculty of Clarkson University, Potsdam, NY, where he currently teaches in the Division of Mathematics and Computer Science. His current research interests include dynamical systems, chaos, control, complex networks, and engineering applications.

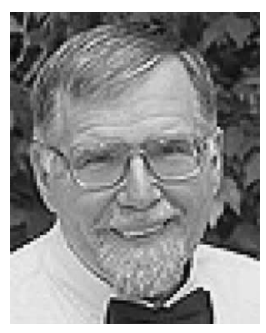

Charles J. Robinson received the B.S. degree in engineering science from Franciscan University of Steubenville, Steubenville, OH, the M.S. degree in electrical engineering from Ohio State University, Columbus, and the D.Sc. degree in electrical engineering from Washington University, Saint Louis, MO.

He was trained as a Biomedical Engineer and a Neuroscientist at Washington University. He was a Postdoctoral Fellow of anesthesiology at Yale University. He is currently the Shulman Chair of Rehabilitation Engineering at Clarkson University, Potsdam, NY, where he is also the Director of the Center for Rehabilitation Engineering, Science and Technology. He is also a Senior Rehabilitation Research Career Scientist (SRRCS) with the U.S. Department of Veterans Affairs, Syracuse, NY, and was the first VA Senior Rehabilitation Research Career Scientist (SRRCS) to be selected in the country. He has been with the VA Research Service for almost 28 years, with postings in Chicago, Pittsburgh, Shreveport, and Syracuse. He taught in bioengineering programs at the University of Illinois-Chicago, the University of Pittsburgh, and Louisiana Tech University, where he was the Watson Eminent Scholar Chair in Biomedical Engineering and Micromanufacturing. He has been a Full Professor in various clinical departments, including Neurology (Loyola), Orthopedic Surgery (Pitt and Louisiana State University Health Sciences Center (LSUHSC)-Shreveport), and Physical Medicine and Rehabilitation, Syracuse, New York [State University of New York (SUNY)] Upstate, Syracuse, and founded and chaired the Department of Rehabilitation Science and Technology at Pitt. 